\title{
In situ observation of ferromagnetic order breaking in MnAs/GaAs(001) and magnetocrystalline anisotropy of $\alpha$-MnAs by electron magnetic chiral dichroism
}

\author{
X. Fu, ${ }^{1}$ B. Warot-Fonrose, ${ }^{1}$ R. Arras, ${ }^{1}$ G. Seine, ${ }^{1}$ D. Demaille, ${ }^{2,3}$ M. Eddrief, ${ }^{2,3}$ V. Etgens, ${ }^{2,3,4}$ and V. Serin ${ }^{1}$ \\ ${ }^{1}$ Université Toulouse, CEMES-CNRS UPR 8011, F-31055 Toulouse, France \\ ${ }^{2}$ Sorbonne Universités, UPMC Université Paris 6, UMR 7588, INSP, 4 Place Jussieu, F-75005 Paris, France \\ ${ }^{3}$ CNRS, UMR 7588, Institut des Nanosciences de Paris, 4 Place Jussieu, F-75005 Paris, France \\ ${ }^{4}$ Institut VeDeCoM - Université de Versailles Saint-Quentin-en-Yvelines, 78000 Versailles, France \\ (Received 2 December 2015; revised manuscript received 15 February 2016; published 11 March 2016)
}

\begin{abstract}
We report an in situ observation of temperature-dependent phase transition in MnAs thin film by transmission electron microscopy (TEM) techniques. Following the identification of the crystallographic transition, from hexagonal $\alpha$-MnAs to quasihexagonal $\beta$-MnAs, the orbital-to-spin moment ratio is measured and a breaking of the ferromagnetic order locally observed, thanks to the electron magnetic chiral dichroism (EMCD) technique. To achieve quantitative information, applying the sum rules to the dichroic signal of magnetic anisotropic materials is accurately discussed. Finally, the orbital-to-spin moment ratio of $\alpha$-MnAs along the easy, hard, and intermediate magnetic axes is estimated by EMCD and compared to implemented density functional theory (DFT) calculations. The influence of the magnetocrystalline anisotropy is locally demonstrated. This work in particular illustrates the feasibility of the EMCD technique for in situ experiments, and proves its potential to explore the anisotropy of magnetic materials.
\end{abstract}

DOI: 10.1103/PhysRevB.93.104410

\section{INTRODUCTION}

MnAs thin films on GaAs(001) substrates have been widely studied in recent years due to their potential use in spintronic applications [1-3]. Thanks to techniques such as scanning tunneling microscopy [4], atomic force microscopy (AFM) [5], and magnetic force microscopy (MFM) [4,5], the temperaturedependent surface topography and the micromagnetic structure have been revealed. The hexagonal $\alpha$-MnAs and the orthorhombic $\beta$-MnAs coexist between approximately $10^{\circ} \mathrm{C}$ and $45^{\circ} \mathrm{C}$ in MnAs thin films with alternating stripes formed along the [0001] direction due to strain stabilization [4]. During the phase transition, the $\alpha$-MnAs gradually transforms into $\beta$-MnAs with temperature rising, until the temperature $T_{c}$ of about $45^{\circ} \mathrm{C}$, where no $\alpha$-MnAs is left [5]. A superconducting quantum interference device (SQUID) has been frequently used to study the magnetization of MnAs thin film. It was reported that $\alpha$-MnAs is ferromagnetic with an easy axis along the [11 20$]$ direction and a hard axis along the [0001] direction within the growth plane (1100) [6]. When the temperature increases, the magnetic anisotropy becomes weaker and the magnetization is simultaneously decreasing [7]. $\mathrm{X}$-ray magnetic circular dichroism (XMCD) was once applied to study local temperature-dependent magnetization on the phase-coexistence region using a probe diameter of $800 \mathrm{~nm}$ [8]. The magnetization versus temperature curve follows the same trend as the one obtained by SQUID, and furthermore, it provides the information of spin and orbital magnetic moment for the exploration of a more fundamental mechanism. It is of interest to further focus on the $\alpha$-MnAs and follow the evolution of local magnetic properties during the phase transition. An emerging technique based on transmission electron microscopy (TEM), electron magnetic chiral dichroism (EMCD) [9], provides a valuable tool to measure local values due to its high spatial resolution, which can be down to 1 $\mathrm{nm}$ [10]. In an ultimate case of tailored beams, the spatial resolution can be even less $[11,12]$.
Electron energy loss spectroscopy (EELS) in the TEM gives access to the unoccupied density of states, and so provides information on electronic structure [13]. In the case of transition metals, $L$-edge spectra correspond to the excitation of a $2 p$ electron to a $3 d$ state, giving access to local magnetic properties which are carried by the $3 d$ electron states. In EMCD, the dichroism signal is the relative difference of such EELS spectra recorded at two chiral positions [9]. These two positions are symmetrical points located on the Thales circle passing through the transmission spot and a Bragg spot on the diffraction pattern as indicated in Fig. 1. At the two specific positions, different combinations of two electron scattering vectors $\mathbf{q}$ and $\mathbf{q}^{\prime}$ give rise to left- and right-handed polarized virtual photons, which are analog to a circular polarized $\mathrm{x}$ ray in XMCD. The magnetic moment information, spin and orbital moments, can be extracted from the dichroism signal by applying sum rules $[14,15]$. Since Schattschneider et al. realized the experiment of magnetic circular dichroism in a TEM in 2006 [9], experimental setups, signal recording methods, data processing methods, and application of sum rules have been constantly improved to make EMCD a more efficient technique for quantitative analysis of magnetic moments [15-20]. The structure of the most commonly investigated materials is cubic, and the anisotropy is still rarely mentioned in papers related to EMCD, contrary to XMCD which is frequently used for that [21].

In this paper we present the in situ temperature-dependent TEM investigation of MnAs thin film, grown on GaAs(001) substrate. The phase transition is observed and controlled from a structural point of view, and EMCD signals recorded. The sum rules are discussed and applied to interpret the signal from $\mathrm{Mn}^{2+}$ in hexagonal $\alpha$-MnAs as well as in the orthorhombic $\beta$-MnAs. A breaking of the ferromagnetic order is observed during the transition. Finally, we explore the magnetic anisotropy along different directions of the $\alpha$-MnAs phase. Magnetic moments measured by EMCD are compared 


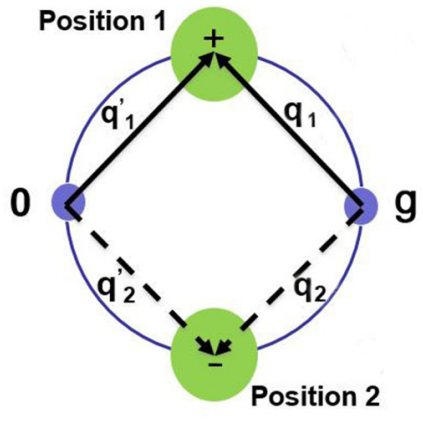

FIG. 1. Positions of the two virtual entrance apertures in the data cube for measurement of EMCD (green disks). The transmission spot $\mathbf{0}$ and diffraction spot $\mathbf{g}$ are represented (blue dots).

to density functional theory (DFT) calculations. This work illustrates the interest of EMCD experiment to dynamically and locally study and quantify magnetization, in particular in the case of ferromagnetic materials with magnetocrystalline anisotropy.

\section{EXPERIMENTAL TECHNIQUES}

The MnAs layers were grown using solid-source molecular beam epitaxy (MBE) on semi-insulating GaAs(001) substrates [4]. The thickness is $150 \mathrm{~nm}$, and the layers are protected by a $10 \mathrm{~nm}$ thick As capping layer. The growth direction of the MnAs layer was at first studied by x-ray diffraction (XRD). XRD $\theta-2 \theta$ scans were carried out on a laboratory 5-circles diffractometer (Rigaku Smartlab), equipped with a $\mathrm{Cu} K_{\alpha 1}$ radiation. MFM was performed to observe the magnetic contrast of the MnAs thin film at remanence after being magnetized along the easy axis. MFM experiment was made on a Bruker Multimode AFM operated in LiftMode procedure.

TEM thin foils were prepared for investigating the crystallographic structure and the local magnetic properties. Planview samples were prepared by polishing from the substrate side until a thickness of $10 \mu \mathrm{m}$, and then finally polished by ion-beam milling. Cross-sectional specimens were also prepared to probe the magnetic signal along different crystallographic axes. TEM studies were performed on a Tecnai F20 microscope operating at $200 \mathrm{kV}$, fitted with an objective lens aberration corrector (CEOS) and imaging filter (Gatan Tridiem). A Gatan 652 double tilt sample holder was used to heat the sample from room temperature (RT) to $50{ }^{\circ} \mathrm{C}$ inside the TEM. In situ observation of the evolution of the crystal structure and local magnetic moment around the transition temperature were achieved. The crystallographic transition of the hexagonal $\alpha$-MnAs to orthorhombic $\beta$-MnAs was followed using the selected area electron diffraction (SAED) technique. The EELS spectra were acquired to check the thickness and elemental composition of the identified area. The EMCD technique was then applied on the same area to acquire dichroic signal at both RT and $50^{\circ} \mathrm{C}$. At RT, the EMCD signal has been acquired along different axes: easy axis [11리, hard axis [0001], and intermediate magnetic axes [11100] of MnAs. This is achieved by using either the plan-view sample, or the cross-section one, tilted or not, taking into account that the magnetic moments are aligned along the optical axis due to the magnetic field from objective lens. At $50^{\circ} \mathrm{C}$, the EMCD signal was acquired on the plan-view sample tilted along the intermediate axis, for comparison with the signal acquired at RT. For EMCD experiment, the crystal was orientated a few degrees away from the zone axis to achieve a two-beam condition for minimization of the $n$-beam dynamic interaction, and we used a large-angle convergent diffraction (LACDIF) setup to obtain a diffraction pattern with two spots in the image plane, using a probe size of about $50 \mathrm{~nm}$ [16]. The spatial resolution was around $50 \mathrm{~nm}$ and the energy resolution is $0.7 \mathrm{eV}$. Energy spectrum imaging (ESI) technique was applied to record a data cube in the energy-loss range of $625-665 \mathrm{eV}$ around the $\mathrm{Mn} L_{2,3}$ edge with an exposure time of $20 \mathrm{~s}$ per energy slit [16]. Both the energy slit width and energy step size are $1 \mathrm{eV}$. Thus the data cube contains both energy loss and scattering angle information. With the ESI technique, the positions of the virtual entrance apertures can be determined accurately during post-process data treatment. It successfully avoids the problem of locating the spectrometer entrance aperture (SEA) or objective aperture (OA), which is tough to be located accurately on the two specific positions. Post corrections of nonisochromaticity and spatial drift were achieved using a home-made Digital-Micrograph image processing package [22]. From the corrected data cube, two EELS spectra were obtained at two specific positions, and the spectra difference which is a dichroic signal was extracted. Then sum rules were applied to the dichroic signal for quantitative analysis, by doing some calculations on the area under the $L_{3}$ curve within $(636-644 \mathrm{eV})$ and the $L_{2}$ curve within (648-656 eV).

To support the experimental result, first-principles calculations based on the DFT and on the generalized gradient approximation (GGA) [23] were performed, using the fullpotential linearized augmented plane waves code Wien2k [24]. The atomic sphere radii were chosen to 2.0 a.u. for both $\mathrm{Mn}$ and As and we made the calculations with the following experimental lattice parameters $a=3.7253 \AA, c=5.7031 \AA$. The irreducible part of the first Brillouin zone was sampled with $1464 k$ points and the convergence parameter RKmax was set to 7.0. The $m_{l}$ and $m_{s}$ moments were calculated for the $d$ electrons of the Mn atom. Spin-orbit interaction has been added in the [1120], [1100], and [0001] direction.

\section{RESULTS AND DISCUSSION}

\section{A. Structural information}

A x-ray diffraction (XRD) $\theta-2 \theta$ scan is shown in Fig. 2. The peaks around $27.7^{\circ}, 57.1^{\circ}$, and $91.7^{\circ}$ correspond to $(1100)$, ( 2200$)$, and ( $\overline{3} 300) \alpha$-MnAs planes, respectively. It indicates that the MnAs film is constituted of only one epitaxial outof-plane orientation. A weak peak denoted by an arrow in the figure, and shifted about $1.3^{\circ}$ from the large ( $\left.\overline{3} 300\right)$ peak of $\alpha$-MnAs, is attributed to $\beta$-MnAs. It indicates the coexistence of the two phases at RT.

As expected from previous studies [4], the MFM scan of Fig. 3(a) confirms that, at room temperature, the MnAs layers display ridges and grooves, alternating $\alpha$ (magnetic) and $\beta$ (nonmagnetic) phases to form a self-organized pattern 

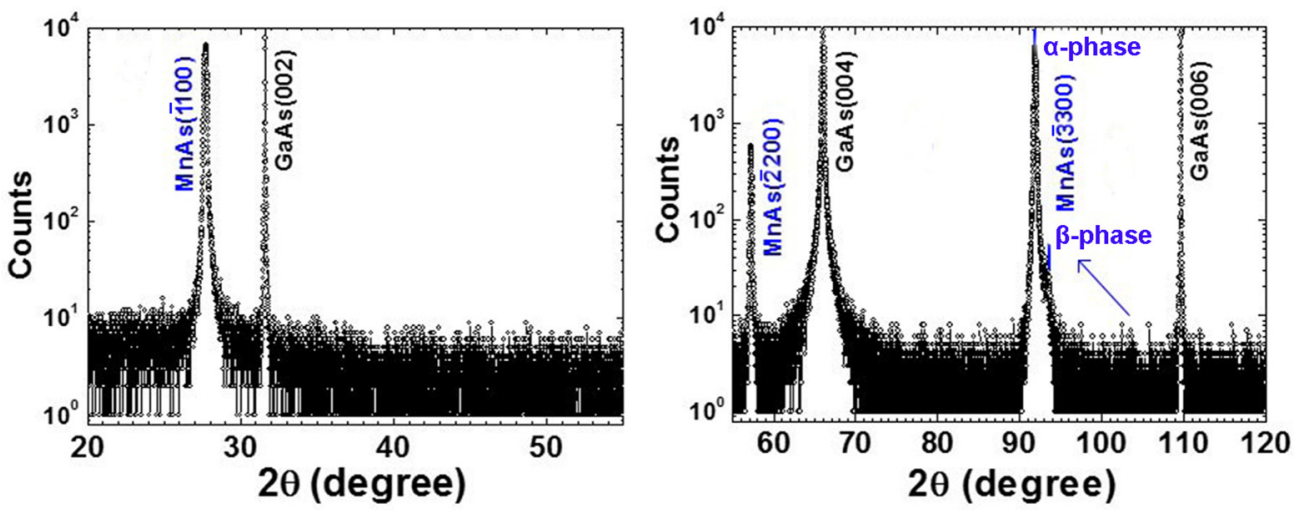

FIG. 2. X-ray diffraction (XRD) $\theta-2 \theta$ scan for MnAs thin film grown on GaAs(001) substrate.

of micron-wide stripes. Figure 3(b) clarifies the relationship between stripe direction and crystal orientation of the $\alpha$-MnAs with respect to the (001) plane of GaAs substrate. The distance between the stripes (about $1 \mu \mathrm{m}$ ) [4] prevents the TEM observation of such a contrast.

In Fig. 4 the high resolution TEM image of the interface, as well as the diffractograms of the different areas (in inset), are shown to demonstrate the epitaxial relationship more clearly. The [11 20 ] easy axis of MnAs is parallel to GaAs [110] and MnAs [0001] is parallel to GaAs [110]. It can be seen that the interface is rather flat, indicating the high-quality epitaxy of MnAs layer on GaAs substrate.

\section{B. In situ observation of phase transition}

\section{Crystallographic transition}

Inside a TEM, the difference between $\alpha$ and $\beta$ phases cannot be easily seen because of their similar atomic structure. For instance, we cannot conclude on the $\alpha$ or $\beta$ structure of the crystal shown in Fig. 4. Nevertheless, it is found that we can differentiate them in the plan-view sample using electron diffraction technique in certain conditions [25]. When the $\alpha$-MnAs is transformed into $\beta$-MnAs, some extra spots appear at specific positions in only some diffraction patterns, demonstrating the crystallographic transition occurred. So, $\alpha$-MnAs has been identified by observing its diffraction pattern along the $[\overline{2} 20 \overline{1}]$ zone axis. On the very same area of identified $\alpha$-MnAs, the crystallographic change upon temperature rise is monitored by observing the evolution of diffraction pattern along the $[\overline{2} 20 \overline{1}]$ zone axis, which corresponds to the [131] zone axis in orthorhombic $\beta$-MnAs.
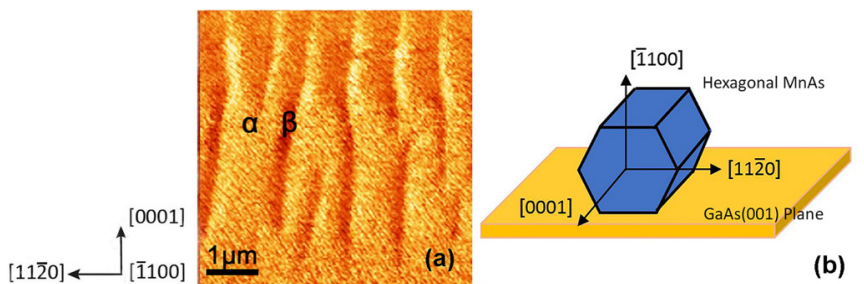

FIG. 3. (a) $5 \times 5 \mu \mathrm{m}^{2}$ MFM scan of MnAs thin film at remanence at RT. (b) Schematic diagram of $\alpha$-MnAs crystal orientation.
Figure 5 demonstrates the evidence of atomic structure evolution during phase transition. The left-hand column shows the images recorded at RT, and at the right those recorded at $50{ }^{\circ} \mathrm{C}$. The selected area is denoted in Figs. 5(a) and 5(d) by white circles indicated by arrows. The thickness of the selected area is measured to be $35 \mathrm{~nm}$. No visible extra spots are observed in the diffraction pattern along the [220 $\overline{1}]$ zone axis in Fig. 5(b), identifying that substantially all the MnAs in the selected area is hexagonal $\alpha$-MnAs at RT. During the temperature increase, from RT to $50^{\circ} \mathrm{C}$, there is a continuous variation of the image contrast in the low-magnification brightfield image. When the sample reaches $40^{\circ} \mathrm{C}$, the previously mentioned extra spots start to be noticeable in the diffraction pattern along $[\overline{2} 20 \overline{1}]$, verifying that the transition to orthorhombic $\beta$-MnAs in the selected area has happened. When the temperature approaches $50^{\circ} \mathrm{C}$, the intensity of the extra spots is stable, and also for the contrast in the low-magnification image. The full duration of the temperature increase up to stabilization is about $5 \mathrm{~min}$. The final low-magnification image and diffraction pattern acquired at $50{ }^{\circ} \mathrm{C}$ are presented in

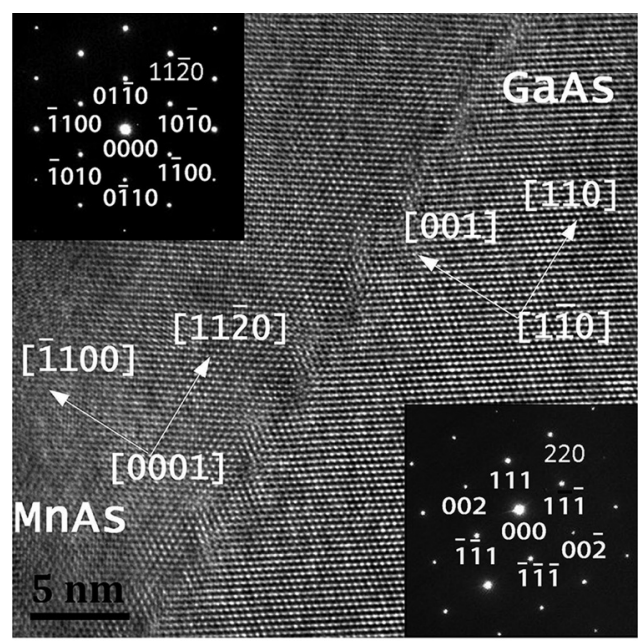

FIG. 4. Cross-sectional HREM image of the heterostructure in GaAs[110] at RT, processed by Wien filter. The upper left inset is the diffraction pattern of MnAs layer acquired at the [0001] zone axis, and in the other inset is the diffraction pattern of GaAs substrate acquired at the $[1 \overline{1} 0]$ zone axis. 
At RT
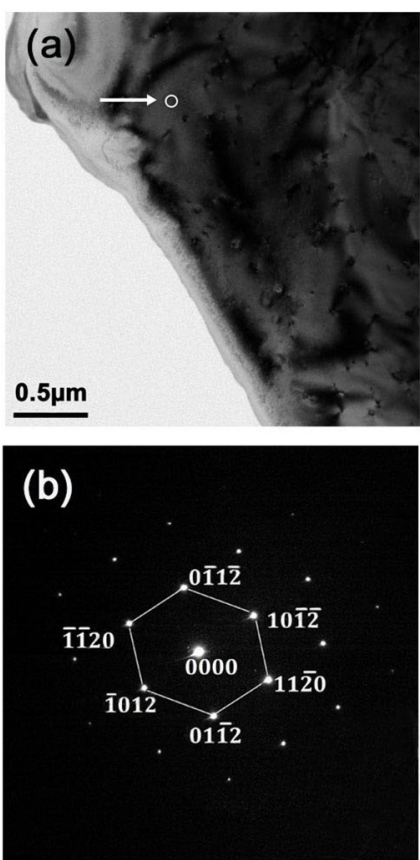

(c)

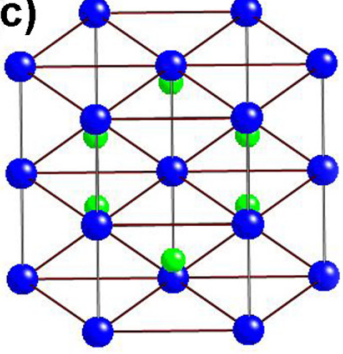

At $50^{\circ} \mathrm{C}$
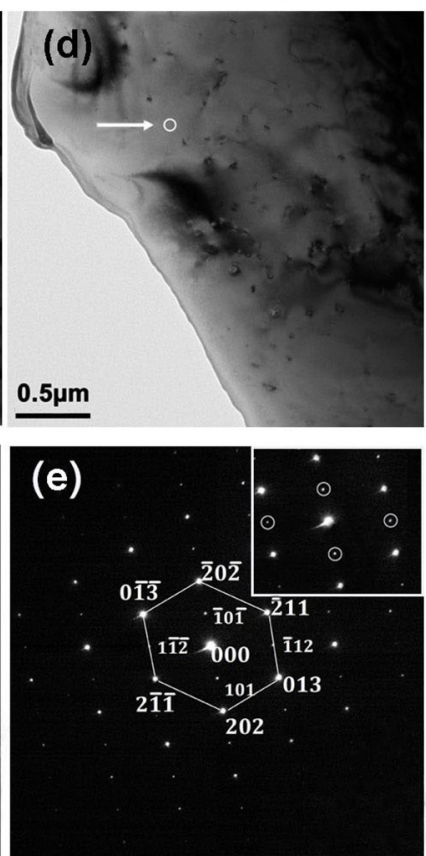

(f)

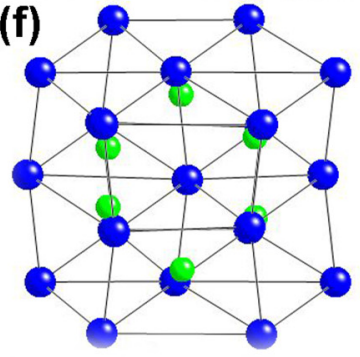

FIG. 5. Low magnification bright-field image of MnAs thin film, (a) at RT, and (d) at $50^{\circ} \mathrm{C}$. (b) Diffraction pattern at RT along the [2201] zone axis, and (c) the corresponding atomic structure seen from the $[\overline{2} 20 \overline{1}]$ view direction. (e) Diffraction pattern at $50^{\circ} \mathrm{C}$ along the [131] zone axis, and (f) the corresponding atomic structure seen from the [ $\overline{13} 1]$ view direction. The blue and bigger dots represent $\mathrm{Mn}$ atoms, and the green and smaller dots represent As atoms.

Figs. 5(d) and 5(e). Substantially, all the $\alpha$-MnAs in the selected area at $50{ }^{\circ} \mathrm{C}$ is assumed to be transformed into $\beta$ phase. Furthermore, it is worth noticing that three possible crystal orientations of $\beta$-MnAs with respect to $\mathrm{GaAs}(001)$ plane have been found after exploring several areas: $\beta$ $\operatorname{MnAs}(001) / / \operatorname{GaAs}(001), \beta-\operatorname{MnAs}(01 \overline{1}) / / \operatorname{GaAs}(001)$, and $\beta$ MnAs (011)//GaAs(001). The diffraction pattern in Fig. 5(e) just belongs to one case which is $\beta$-MnAs $(01 \overline{1}) / / \operatorname{GaAs}(001)$. In the other cases, extra spots appear at different specific positions, but similarly exhibit the phase change. This information is in agreement with the XRD results. The $2 \theta$ angles of the (006), (03) $)$, and (033) planes corresponding to the three possible orientations of $\beta$-MnAs are very close and cannot be discriminated in the $\mathrm{x}$-ray $\theta-2 \theta$ diagram. So, one peak representative of the three possible orientations of $\beta$-MnAs is present as a shoulder of the intense peak of $\alpha$-MnAs, mainly present at RT.

At RT
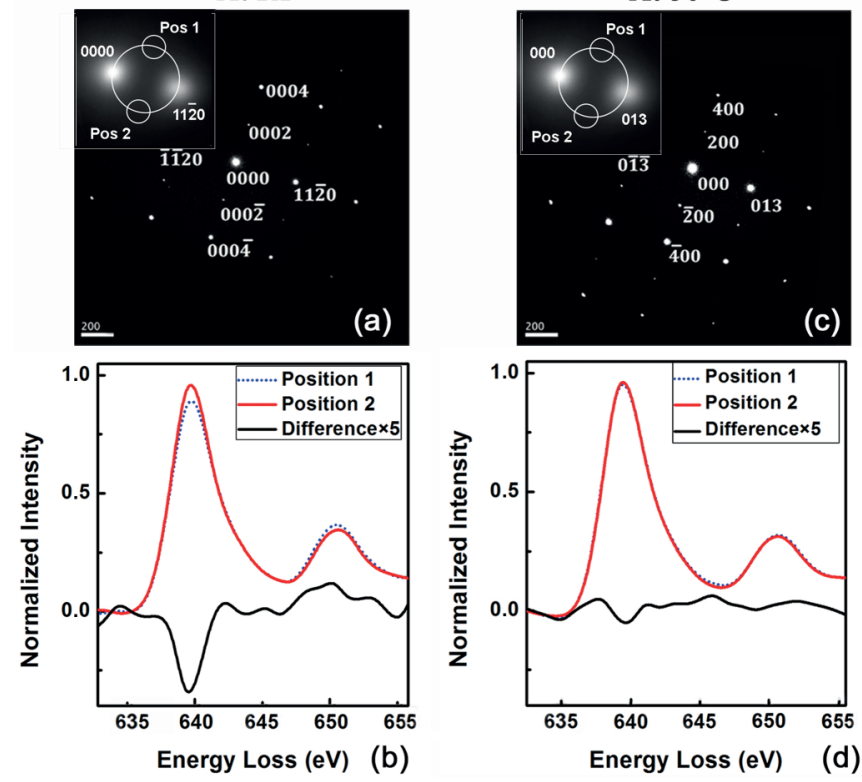

FIG. 6. Diffraction pattern along the zone axis of (a) [11100] at RT and (c) $[0 \overline{3} 1]$ at $50{ }^{\circ} \mathrm{C}$. The insets in (a) and (c) are the energy filtered (energy loss from 625-665 eV around the $\mathrm{Mn} L_{2,3}$ edges) diffraction patterns in two-beam condition. EELS spectra are acquired at position 1 and 2 as well as the spectra difference multiplied by 5 (b) at RT, and (d) at $50{ }^{\circ} \mathrm{C}$. A low pass filter has been applied to the spectra for better visibility.

\section{Temperature-dependent EMCD signal}

The EMCD results are demonstrated in Fig. 6. First, the similarity of the two diffraction patterns is obviously seen in Figs. 6(a) and 6(c). In this experiment, the $\alpha$-MnAs crystal at RT is orientated along the zone axis of [11100] [Fig. 6(a)], and then tilted several degrees to reach two-beam configuration where only diffraction spot $(11 \overline{2} 0)$ is excited. At $50^{\circ} \mathrm{C}$, the transformed $\beta$-MnAs crystal is orientated along the zone axis [031] [Fig. 6(c)], which is parallel to the $\alpha$-MnAs [ $\overline{1} 100]$ direction, and then reaches the same two-beam condition where only diffraction spot (013) is excited. Thus, the same diffraction spot is selected as shown in the insets. The dynamic interaction of the electron beam in the two cases are very analogous, thus it can be excluded as an influence factor when comparing dichroism signals obtained at RT and $50^{\circ} \mathrm{C}$. Figure 6(b) shows an obvious dichroism signal at RT, while the dichroism signal disappears after the phase transition. Sum rules have then been applied to quantitatively analyze the change in the magnetic moment with phase transition.

For $3 d$ transition metal ions or atoms like $\mathrm{Mn}^{2+}$, the EMCD sum rule is written as Eq. (1) [14], $\sigma_{2}-\sigma_{1}$ is the spectra difference

$$
\begin{gathered}
\frac{\int_{L_{3}}\left(\sigma_{2}-\sigma_{1}\right) d E-2 \int_{L_{2}}\left(\sigma_{2}-\sigma_{1}\right) d E}{\int_{L_{2}+L_{3}}\left(\sigma_{2}-\sigma_{1}\right) d E} \\
=\frac{4\left\langle S_{z}\right\rangle+14\left\langle T_{z}\right\rangle}{3\left\langle L_{z}\right\rangle} \approx \frac{2 m_{s}}{3 m_{l}} .
\end{gathered}
$$

The expression $\int_{L_{3}}\left(\sigma_{2}-\sigma_{1}\right) d E$ means the integrated value of the difference spectrum within the energy-loss range corre- 
sponding to the $L_{3}$ peak. $\left\langle L_{z}\right\rangle,\left\langle S_{z}\right\rangle$, and $\left\langle T_{z}\right\rangle$ are, respectively, the ground-state values of orbital momentum, spin momentum, and magnetic dipole operators projected along the $z$ direction. $m_{l}$ and $m_{s}$ are, respectively, the corresponding orbital and spin magnetic moment, expressed as $m_{l}=\left\langle L_{z}\right\rangle \mu_{B} / \hbar$ and $m_{s}=2\left\langle S_{z}\right\rangle \mu_{B} / \hbar$. In this equation, $\left\langle T_{z}\right\rangle$ is negligible compared to $\left\langle S_{z}\right\rangle$ for $3 d$ transition atoms [26]. It is worth noting that an assumption of importance has been made to simplify the derivation of sum rules: all the magnetic moments in the detected sample must be aligned along the $z$ axis, which is perpendicular to the plane defined by the diffusion vectors $\mathbf{q}$ and $\mathbf{q}^{\prime}$ (cf. Fig. 1). In standard TEM operating modes, the sample is under an external magnetic field as large as $2 \mathrm{~T}$, due to the objective lens, so that the magnetic moments should be aligned along the magnetic field. The magnetic field is parallel to the optical axis, which is also the $z$ axis mentioned above. If the precondition is not met, what is obtained with sum rules is just a projection of magnetic moment on the $z$ axis.

For the ferromagnetic $\alpha-\mathrm{MnAs}$, it is easy to achieve magnetic saturation in TEM, since the saturation field along the easy axis is less than $100 \mathrm{mT}[6,7]$. However in practice, the easy axis of the film is not necessarily parallel to the magnetic field in TEM, but always tilted away from it to reach a two-beam condition. So it is of help to understand magnetic anisotropy field of $\mathrm{MnAs} / \mathrm{GaAs}(001)$ thin film and determine the applied magnetic field required to saturate the sample along any axis.

As mentioned before, the MnAs film on GaAs(001) substrate has a magnetic hard axis along [0001], and an easy axis along [11 $\overline{2} 0]$ in the growth plane (1100). The magnetocrystalline anisotropy is the major contribution to the in-plane magnetic anisotropy. Thus, the in-plane magnetic anisotropy field $H_{k}$ can be described, according to Refs. [8,27], as $H_{k}=-2\left(K_{1}+2 K_{2}\right) / \mu_{0} M_{s}$, where $K_{1}$ and $K_{2}$ is the first and second order of anisotropy constant, respectively, $\mu_{0}$ is vacuum permeability, and $M_{s}$ is the saturation magnetization. The values of $K_{1}=-7.9 \times 10^{5} \mathrm{~J} / \mathrm{m}^{3}, K_{2}=1.1 \times 10^{5} \mathrm{~J} / \mathrm{m}^{3}$, and $M_{s}=0.65 \times 10^{6} \mathrm{~A} / \mathrm{m}$ around RT [8] were used to estimate $B_{k}=H_{k} \mu_{0}=1.75 \mathrm{~T}$. The reported values of in-plane magnetic anisotropy field of $2 \mathrm{~T}[7,28,29]$ is consistent with our calculated value. The film also has an out-of-plane magnetic anisotropy, but weaker than the in-plane one [7,29], which means the saturation field along the [0001] hard axis is larger than that along the out-of-plane $[\overline{1} 100]$ intermediate axis. The magnetic field from the objective lens being measured is $2 \mathrm{~T}$ in our experimental conditions, it is high enough to saturate the sample regardless of the sample orientations inside the TEM. We then apply the sum rule to the signal shown in Fig. 6(b) but without being filtered, and the $m_{l} / m_{s}$ ratio deduced is of $0.08 \pm 0.04$. More detailed explanations will be given in the next section.

In the case of $\beta$-MnAs, it is more complicated. There is even no consensus on its magnetic nature yet. Theoretical and experimental evidences can be found to support both paramagnetism and antiferromagnetism [30]. If it is paramagnetic, under the magnetic field of $2 \mathrm{~T}$ in TEM, the disorderly arranged magnetic moments have the tendency to align along the $z$ axis. Note that there is intrinsic net magnetic moment of $\mathrm{Mn}$ atom, and the magnetization projected along the $z$ axis is not zero under magnetic field, therefore the dichroism signal $\sigma_{2}-\sigma_{1}$ should theoretically appear, according to Eq. (2), where $N_{h}$ is the number of holes in the $d$ bands and $K$ contains all the information related to the dynamical diffraction effects [13]

$$
\left\langle S_{z}\right\rangle=\frac{3 N_{h}}{2 K} \frac{\int_{L_{3}}\left(\sigma_{2}-\sigma_{1}\right) d E-2 \int_{L_{2}}\left(\sigma_{2}-\sigma_{1}\right) d E}{\int_{L_{2}+L_{3}}\left(\sigma_{2}+\sigma_{1}\right) d E} .
$$

But no dichroism signal appearing in Fig. 6(b) is sufficient evidence to reject its paramagnetism, because the magnetization of $\beta$-MnAs under an applied magnetic field of 2 $\mathrm{T}$ is much smaller than the one of $\alpha$-MnAs. In Ref. [28] the magnetization of $\beta$-MnAs around RT is measured to be almost 10 times smaller than that of $\alpha$-MnAs under $2 \mathrm{~T}$. Thus, the dichroism signal of paramagnetic $\beta$-MnAs may be too weak to be detected by EMCD technique, due to the insufficient signal-to-noise ratio. On the other side, if $\beta$-MnAs is antiferromagnetic, the magnetization is even smaller, and the dichroism signal is still more difficult to obtain. Another possible explanation for antiferromagnetic behavior is that the dichroism signal from the parallel moments will be in opposite sign with the signal from the antiparallel moments. If the total moments are zero, their contribution to the dichroism signal will cancel each other out. With the disappearance or largely weakening of the dichroism signal, the ferromagnetic order is seen to be broken following the crystallography transition.

\section{Anisotropic EMCD signal for magnetocrystalline anisotropic $\alpha$-MnAs}

In the previous section, it is mentioned that $\alpha$-MnAs thin layer on $\mathrm{GaAs}(001)$ substrate has a large magnetocrystalline anisotropy. The external magnetic field of $2 \mathrm{~T}$ is enough to saturate the sample along the hard axis [0001] at RT, but only $1 \mathrm{~T}$ along the intermediate axis [11100] [29] and $100 \mathrm{mT}$ along the easy axis [11 20$]$ are needed to saturate the layer. In principle, the spin moments are nearly isotropic and all of them can be aligned when the sample is magnetically saturated along the $z$ axis in the TEM, but there is still charge anisotropy of the valence states involved in the core excitation process through the spin-orbit coupling [31]. In hexagonal MnAs, the orbital angular momentum of $\mathrm{Mn}^{2+}$ is partly frozen due to the crystal field. The projection of orbital momentum $\left\langle L_{z}\right\rangle$ varies with the sample orientation under the fixed magnetic field along the $z$ axis. As a result, the measured EMCD signal and the obtained moment ratio cannot be free from the sample orientation, but are anisotropic.

Figure 7 shows the result of EMCD experiment performed on the cross sectional sample orientated along the direction close to the hard axis [0001]. This direction is perpendicular to the intermediate axis and the spot (1010) is selected in Fig. 7(a). Figure 7(b) displays the EMCD signal. We then apply the sum rule to the signal without being filtered, and the $m_{l} / m_{s}$ ratio is of $0.06 \pm 0.04$. When compared with the experimental value of $0.08 \pm 0.04$ obtained around intermediate axis [1100], it is found that the ratio along the axis around the hard axis is obviously lower. In addition, the experimental value obtained around easy axis $[11 \overline{2} 0]$ is measured to be $0.09 \pm 0.04$, which is 0.01 larger than the value obtained around the intermediate axis. Correspondingly, the DFT calculations also give different values in different situations: $m_{l} / m_{s}=0.008$ when the spin 

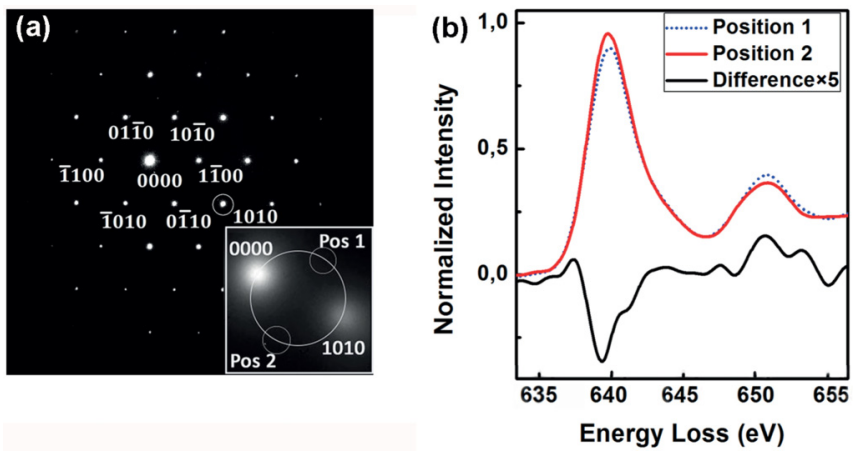

FIG. 7. (a) Diffraction pattern along the zone axis of [0001] at RT, in inset: energy filtered diffraction pattern in two-beam condition. (b) EELS spectra at RT from position 1 and 2 as well as spectra difference multiplied by 5 . A low pass filter has been applied to the spectra for better visibility.

and orbital moments are coupled along the easy axis; $m_{l} / m_{s}=$ 0.005 when along the hard axis; and $m_{l} / m_{s}=0.008$ when along the intermediate axis. This information is gathered in Table I, where the XMCD experimental value of $0.03 \pm 0.02$ obtained along the easy axis at around RT is extracted from Ref. [8]. From Table I it can be seen that the experimental EMCD value of $0.09 \pm 0.04$ for easy axis measurement is larger than the XMCD value of $0.03 \pm 0.01$ obtained along the same axis. Data correction of spin moment with a constant of 1.47 has been proceeded to compensate for the effect of the mixing between $L_{2}$ and $L_{3}$ edges for the XMCD result and not for EMCD ones. The experimental measurements, in particular when compared to the theoretical one, can be considered to be of the same order of magnitude, largely far from the theoretical ones. The discrepancy between the experimental and theoretical results may partly originate from the simplification of the theoretical calculation. The theoretical calculations are based on the atomic ground state at $T=0 \mathrm{~K}$, and are performed for $d$ electrons inside atomic spheres only, whereas the experiment was made at RT where other excited states can mix into the ground state due to thermal motion. In addition, the sample is orientated a few degrees away from the zone axis to a two-beam condition in the EMCD experiment, so the spin-orbit coupling is not exactly the same as set in the theoretical calculation. Taking into account these weaknesses, relative EMCD variations observed were expected from the predicted ones. So we can say that the EMCD technique is an interesting technique to study anisotropy of ferromagnetic material. That is to say, if the diffraction spot in the two-beam condition is selected from a different diffraction pattern due to different sample orientation,
TABLE I. Comparison of the orbital-to-spin moment ratio deduced from sum rules along the easy, hard, and intermediate $\alpha$-MnAs axes obtained with EMCD and XMCD technique [8], and DFT calculations are also provided. EMCD values along the hard and easy axes are obtained in TEM cross-section configuration and that obtained along the intermediate axis is in plan-view one. The thickness of the investigated area for measurement along hard, intermediate, and easy axes is 30,35 , and $37 \mathrm{~nm}$, respectively. The errors on the EMCD values come from different main factors: noise, post edge normalization, plural scattering, shape, and position of apertures in Thales circle [17,18,32,33]. It is estimated here from the standard deviation on the treatment of 5-10 less noisy selected data cubes for each orientation.

\begin{tabular}{llcl}
\hline \hline & $\begin{array}{l}\text { Hard axis } \\
{[0001]}\end{array}$ & $\begin{array}{l}\text { Intermediate axis } \\
{[\overline{1} 100]}\end{array}$ & $\begin{array}{l}\text { Easy axis } \\
{[11 \overline{2} 0]}\end{array}$ \\
\hline EMCD & $0.06 \pm 0.04$ & $0.08 \pm 0.04$ & $0.09 \pm 0.04$ \\
XMCD & - & - & $0.03 \pm 0.01^{\mathrm{a}}$ \\
DFT & 0.005 & 0.008 & 0.008 \\
\hline \hline
\end{tabular}

${ }^{\mathrm{a}}$ Reference [8].

the measured ratio may change. So making clear the sample orientation or the selected diffraction spot is necessary to understand the measured dichroism signal and the moment information it carries.

\section{CONCLUSION}

In conclusion, we presented in situ observation of the phase transition in MnAs thin film when the film was heated from RT to $50^{\circ} \mathrm{C}$ in TEM. The phase transition from $\alpha$-MnAs to $\beta$-MnAs was verified by the crystallographic transition from a hexagonal structure to an orthorhombic one, and the EMCD signal acquired before and after crystallographic transition shows the breaking of the ferromagnetic order during phase transition. We discussed the application of EMCD sum rules to the dichroism signal obtained from hexagonal ferromagnetic $\alpha$-MnAs, and verified that the EMCD signal and the measured magnetic moment ratio are anisotropic for the magnetic anisotropic materials.

\section{ACKNOWLEDGMENTS}

This work is supported by the French national project EMMA (ANR12 BS10 013 01) and by the European Union under the Seventh Framework Program under a contract for an Integrated Infrastructure Initiative Reference 312483ESTEEM2. The authors of this work were granted access to the HPC resources of CALMIP under the allocations P1509. The authors would like to thank S. Cherifi for fruitful discussions and C. Crestou for her valuable help in the sample preparation.
[1] M. Ramsteiner, H. Y. Hao, A. Kawaharazuka, H. J. Zhu, M. Kästner, R. Hey, L. Däweritz, H. T. Grahn, and K. H. Ploog, Phys. Rev. B 66, 081304 (2002).

[2] V. Garcia, H. Jaffrès, M. Eddrief, M. Marangolo, V. H. Etgens, and J. M. George, Phys. Rev. B 72, 081303 (2005).
[3] C. Pampuch, A. K. Das, A. Ney, L. Däweritz, R. Koch, and K. H. Ploog, Phys. Rev. Lett. 91, 147203 (2003).

[4] R. Breitwieser, F. Vidal, I. L. Graff, M. Marangolo, M. Eddrief, J.-C. Boulliard, and V. H. Etgens, Phys. Rev. B 80, 045403 (2009). 
[5] J. Kim, H. Akinaga, and J. Kim, Appl. Phys. Lett. 98, 102511 (2011).

[6] K.-S. Ryu, J. Kim, Y. Lee, H. Akinaga, T. Manago, R. Viswan, and S.-C. Shin, Appl. Phys. Lett. 92, 082503 (2008).

[7] V. Z. C. Paes, I. L. Graff, J. Varalda, V. H. Etgens, and D. H. Mosca, J. Phys.: Condens. Matter 25, 046003 (2013).

[8] J. M. Wikberg, R. Knut, S. Bhandary, I. di Marco, M. Ottosson, J. Sadowski, B. Sanyal, P. Palmgren, C. W. Tai, O. Eriksson, O. Karis, and P. Svedlindh, Phys. Rev. B 83, 024417 (2011).

[9] P. Schattschneider, S. Rubino, C. Hébert, J. Rusz, J. Kuneš, P. Novák, E. Carlino, M. Fabrizioli, G. Panaccione, and G. Rossi, Nature (London) 441, 486 (2006).

[10] J. Salafranca, J. Gazquez, N. Pérez, A. Labarta, S. T. Pantelides, S. J. Pennycook, X. Batlle, and M. Varela, Nano Lett. 12, 2499 (2012).

[11] D. Pohl, S. Schneider, J. Rusz, and B. Rellinghaus, Ultramicroscopy 150, 16 (2015).

[12] P. Schattschneider, I. Ennen, M. Stoger-Pollach, J. Verbeeck, V. Mauchamp, and M. Jaouen, Ultramicroscopy 110, 1038 (2010).

[13] P. E. Batson, Nature (London) 366, 727 (1993).

[14] L. Calmels, F. Houdellier, B. Warot-Fonrose, C. Gatel, M. J. Hÿtch, V. Serin, E. Snoeck, and P. Schattschneider, Phys. Rev. B 76, 060409 (2007).

[15] J. Rusz, O. Eriksson, P. Novák, and P. M. Oppeneer, Phys. Rev. B 76, 060408 (2007).

[16] B. Warot-Fonrose, F. Houdellier, M. J. Hÿtch, L. Calmels, V. Serin, and E. Snoeck, Ultramicroscopy 108, 393 (2008).

[17] S. Muto, J. Rusz, K. Tatsumi, R. Adam, S. Arai, V. Kocevski, P. M. Oppeneer, D. E. Bürgler, and C. M. Schneider, Nat. Commun. 5, 3138 (2014).

[18] J. Rusz, H. Lidbaum, S. Rubino, B. Hjörvarsson, P. M. Oppeneer, O. Eriksson, and K. Leifer, Phys. Rev. B 83, 132402 (2011).

[19] B. Warot-Fonrose, C. Gatel, L. Calmels, V. Serin, E. Snoeck, and S. Cherifi. J. Appl. Phys. 107, 09D301 (2010).
[20] T. Thersleff, J. Rusz, S. Rubino, B. Hjörvarsson, Y. Ito, N. Zaluzec, and K. Leifer, Sci. Rep. 5, 13012 (2015).

[21] G. van der Laan and A. I. Figueroa, Coord. Chem. Rev. 277-278, 95 (2014).

[22] C. Gatel, B. Warot-Fonrose, and P. Schattschneider, Ultramicroscopy 109, 1465 (2009).

[23] J.P. Perdew, K. Burke, and M. Ernzerhof, Phys. Rev. Lett. 77, 3865 (1996).

[24] P. Blaha, K. Schwarz, G. K. H. Madsen, D. Kvasnicka, and J. Luitz, WIEN2K, An Augmented Plane Wave + Local Orbitals Program for Calculating Crystal Properties (Karlheinz Schwarz, Technische Universität Wien, Austria, 2001).

[25] X. Fu, B. Warot-Fonrose, R. Arras, D. Demaille, M. Eddrief, V. Etgens, and V. Serin, Appl. Phys. Lett. 107, 062402 (2015).

[26] P. Carra, B. T. Thole, M. Altarelli, and X. Wang, Phys. Rev. Lett. 70, 694 (1993).

[27] K. H. J. Buschow and F. R. de Boer, Physics of Magnetism and Magnetic Materials (Kluwer Academic/Plenum, New York, 2003).

[28] A. Ney, T. Hesjedal, C. Pampuch, J. Mohanty, A. K. Das, L. Däweritz, R. Koch, and K. H. Ploog, Appl. Phys. Lett. 83, 2850 (2003).

[29] T. Manago, H. Kuramochi, and H. Akinaga, Surf. Sci. 600, 4155 (2006).

[30] H. Yamaguchi, A. K. Das, A. Ney, T. Hesjedal, C. Pampuch, D. M. Schaadt, and R. Koch, Europhys. Lett. 72, 479 (2005).

[31] P. A. van Aken and S. Lauterbach, Phys. Chem. Minerals 30, 469 (2003).

[32] S. Muto, K. Tatsumi, and J. Rusz, Ultramicroscopy 125, 89 (2013).

[33] Z. Q. Wang, X. Y. Zhong, R. Yu, Z. Y. Cheng, and J. Zhu, Nat. Commun. 4, 1395 (2013). 